\title{
Diabetes Mellitus as a Predictor of Retinopathy Associated With Acute Myocardial Infarction
}

\author{
Takuji Katayama, MD*; Takanori Yasu, MD*,†; Nozomi Kinoshita, MD**; \\ Akihiro Kakehashi, MD**; Norifumi Kubo, MD*; Muneyasu Saito, MD*; \\ Shin-ichi Momomura, MD*; Masanobu Kawakami, MD*
}

\begin{abstract}
Background: A unique transient retinopathy characterized by soft exudates around the optic disc after percutaneous coronary intervention (PCI) for acute myocardial infarction (AMI) has been reported, so in the present study the risk factors for retinopathy associated with AMI (RAMI) were investigated.

Methods and Results: The study group comprised 62 patients with their first AMI who underwent successful PCI within $24 \mathrm{~h}$ of onset (48 men, 14 women; age $63 \pm 10$ years). The fundus of each eye was assessed on days 3-5, and again at 4 weeks after AMI onset. New soft exudates developed in 29 patients (47\%) at 4 weeks. The frequency of diabetes mellitus (DM), and the hemoglobin Alc and peak creatine kinase concentrations were higher in patients with than in those without RAMI (55\% vs $21 \%, \mathrm{P}=0.008 ; 7.0 \pm 2.0 \%$ vs $5.9 \pm 1.4 \%, \mathrm{P}=0.013$; and 3,428 $\pm 2,210 \mathrm{IU} / \mathrm{L}$ vs $2,352 \pm 1,652 \mathrm{IU} / \mathrm{L}, \mathrm{P}=0.036$, respectively). Multivariate analysis identified $\mathrm{DM}$ as an independent predictive factor for the occurrence of RAMI (odds ratio, 6.60; 95\% confidence interval, 1.68-25.90; $\mathrm{P}=0.007$ ).
\end{abstract}

Conclusions: DM might be a risk factor for RAMI. (Circ J 2009; 73: 1278-1282)

Key Words: Acute myocardial infarction; Diabetes mellitus; Ischemia; Microcirculation; Reperfusion

$\mathbf{I}$ $\mathrm{n}$ acute myocardial infarction (AMI), ischemia-reperfusion causes local and even systemic inflammation through various mechanisms!-3 Clinically adverse manifestations that might be associated with systemic inflammation in AMI include systemic inflammatory response syndrome $^{4}$ and acute respiratory distress syndrome ${ }^{5}$ accompanied by hemodynamic collapse. However, little is understood about remote organ disorders associated with AMI.

A few recent studies, including our own, have shown that a transient retinopathy develops in patients after reperfusion therapy for AMI6-8 Retinopathy associated with AMI (RAMI) is characterized by soft exudates (cotton wool spots) around the optic disc with or without superficial hemorrhage that appear 2-8 weeks after the onset of AMI and disappear without any specific treatment. In our previous study, none of the patients with RAMI experienced impaired visual acuity, although a few patients reported visual complaints?, 8 The condition developed in approximately half of patients with a first episode AMI who underwent reperfusion therapy within $24 \mathrm{~h}$ of onset? However, details of the mechanism and risk factors for this unique retinopathy remain unknown. The present study investigated major predictors of RAMI

(Received August 13, 2008; revised manuscript received January 22, 2009; accepted February 10, 2009; released online April 28, 2009)

*First Department of Integrated Medicine, **Division of Ophthalmology, Saitama Medical Center, Jichi Medical University, Saitama and Department of Clinical Pharmacology and Therapeutics, Graduate School of Medicine, University of the Ryukyus, Okinawa, Japan An abstract of this work was presented at the Annual Scientific Session of the American Heart Association on November 15, 2006.

Mailing address: Takanori Yasu, MD, Department of Clinical Pharmacology and Therapeutics, Graduate School of Medicine, University of the Ryukyus, 207 Uehara, Nishihara, Okinawa 903-0215, Japan. E-mail: tyasu@shintoshin.nir.jp

All rights are reserved to the Japanese Circulation Society. For permissions, please e-mail: cj@j-circ.or.jp and the impact of RAMI on long-term prognosis.

\section{Methods}

\section{Study Patients}

We identified 70 consecutive patients with first AMI admitted to the coronary care unit between May 2001 and January 2002, and between October 2004 and February 2005. All patients underwent percutaneous coronary intervention (PCI) within $24 \mathrm{~h}$ after the onset of AMI (Figure 1). We excluded 1 patient with a cardiac rupture 2 days after PCI, 3 patients who refused ophthalmic fundus examination and 4 patients for whom fundus photographs were inadequate for evaluation because of cataracts or small pupils, leaving 62 patients (48 men, 14 women; average age, 63 years; range, 39-85 years) (Figure 1). Written informed consent was given by all patients before ophthalmic fundus examination.

\section{Protocol}

The Ethics Committee of Jichi Medical School approved the single-center prospective study protocol. Our previous studies showed that the soft exudates with or without superficial hemorrhage that characterize RAMI appear from 2 weeks after AMI onset and peak at 4 weeks? Based on those results, non-mydriatic funduscopy or fundus photography with a fundus camera (Nonmyd $\alpha$-D; Kowa, Nagoya, Japan) was performed by an ophthalmologist (N.K.) 3-5 days after AMI onset to evaluate the baseline status of both eyes; 28 patients had funduscopy only without photos at baseline. Fundus photography was performed 4 weeks after AMI onset using the same fundus camera for both eyes. At the same time, all patients were interviewed by attending physicians of the cardiovascular division or division of ophthalmology regarding neurological symptoms and visual disturbances 


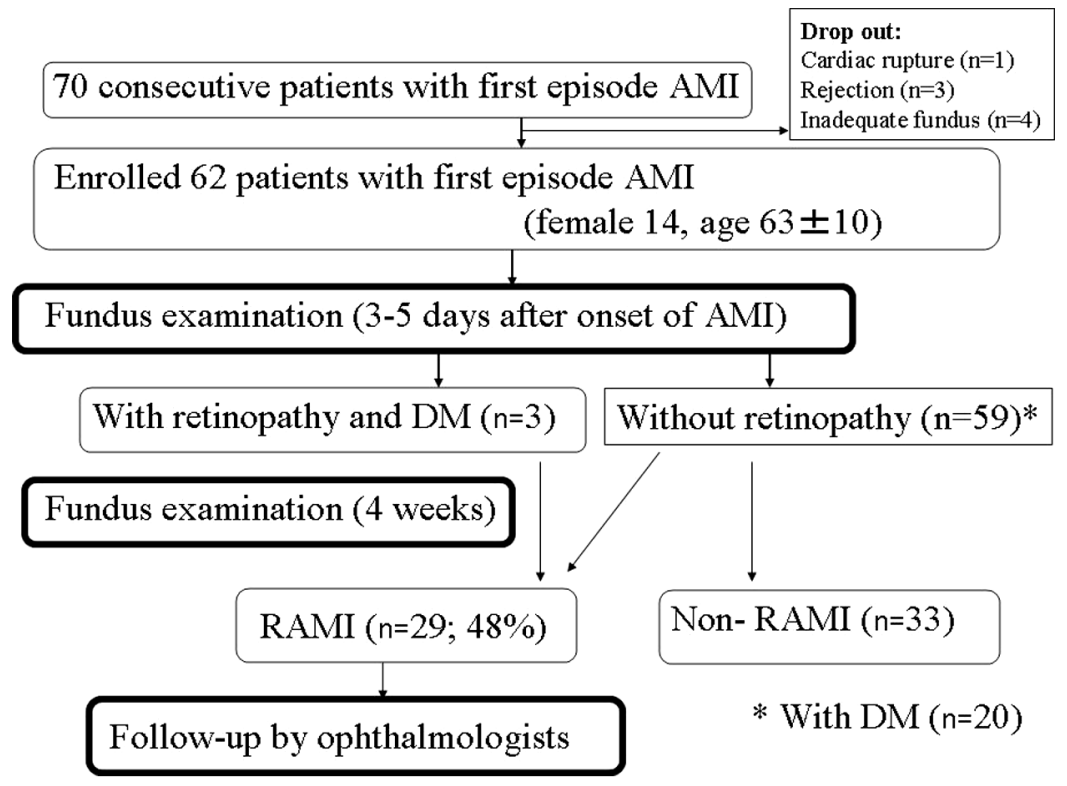

Figure 1. Study design and overall results. Study patients were 62 of 70 consecutive patients with a first acute myocardial infarction (AMI). First fundus examination 3-5 days after the onset of AMI demonstrated diabetic retinopathy in 3 patients but not in the other 59 patients. In the second fundus examination, all 3 patients who had diabetic retinopathy in the first examination showed new soft exudates, and 33 of the 59 patients who did not have diabetic retinopathy in the first examination showed new soft exudates. DM, diabetes mellitus; RAMI, retinopathy associated with AMI. such as blurred vision. Two ophthalmologists (N.K., A.K.) evaluated all fundus photographs, and were unaware of both the patient assignment and whether the photographs were from the initial or follow-up examination. Patients with new soft exudates and/or new hemorrhages by 4 weeks after AMI onset were diagnosed as having RAMI and were periodically observed by ophthalmologists. According to our previous reports, RAMI can be easily differentiated from diabetic retinopathy by the absence of other changes that commonly coexist with cotton wool spots. Diabetic retinopathy is the most common etiology of cotton wool spots, and capillary microaneurysms, dot and blot hemorrhages, and hard exudates usually precede the appearance of such spots.

All patients received oral aspirin $(162 \mathrm{mg})$ immediately after admission and intravenous heparin (10,000 units) before PCI. Tissue plasminogen activator, an aspiration catheter for thrombectomy, and a balloon and stent were used at the discretion of the operator during PCI. Left ventriculography was performed to determine wall motion abnormalities and left ventricular ejection fraction after PCI. Heparin was continued as an intravenous infusion (15,000-20,000 units/day) for the next $72 \mathrm{~h}$ and adjusted to achieve a target activated partial thromboplastin time of 2.0- to 3.0-fold the control level. Nicorandil was also administered as a continuous infusion ( $4 \mathrm{mg} / \mathrm{h} \mathrm{IV}$ ) for $72 \mathrm{~h}$ after admission? All patients received aspirin $(81 \mathrm{mg} /$ day) and all those with implanted bare metal stents also received ticlopidine $(200 \mathrm{mg} /$ day $)$ for 4 weeks after PCI. Patients with atrial fibrillation or left ventricular aneurysm were given warfarin. Clinical reperfusion injury was defined as additional ST-segment elevation without coronary flow reduction during reperfusion therapy. Pre-infarction angina was defined as an attack within $48 \mathrm{~h}$ before AMI onset. Slow flow during PCI was defined as a decrease in Thrombolysis In Myocardial Infarction flow grade $^{10}$ by more than 1 grade compared with the grade before balloon dilatation or stent implantation without evidence of dissection, severe stenosis or vasospasm.

Blood samples were collected from each patient on admission and on each subsequent day until 4 days after admission to perform blood chemistry tests. The creatine kinase $(\mathrm{CK})$ concentration was routinely measured every $4 \mathrm{~h}$ after admission to identify the peak value. Hemoglobin
(Hb) A1c and total cholesterol levels were also measured on admission. Levels of C-reactive protein (CRP) were measured by monoclonal antibody in an assay using latex that enabled measurement over a wide range $(0.01-42 \mathrm{mg} / \mathrm{dl})$ of concentrations (NANOPIA CRP; Daiichi Pure Chemical, Tokyo, Japan). Normal values for CRP are $<0.2 \mathrm{mg} / \mathrm{dl}{ }^{11}$

Coronary risk factors were identified from medical history or hospital data. Patients were regarded as having diabetes mellitus (DM), hypercholesterolemia or hypertension when a history of these was elicited or the patient had been on a diet or pharmacotherapy for these conditions. In addition, DM was diagnosed for HbAlc levels $\geq 6.5 \%$, and hypercholesterolemia was diagnosed if the total cholesterol level was $\geq 220 \mathrm{mg} / \mathrm{dl}$ on admission. Hypertension was diagnosed when systolic blood pressure was $\geq 140 \mathrm{mmHg}$ and/or diastolic blood pressure was $\geq 90 \mathrm{mmHg}$ in hospital.

Follow-up study was performed by reviewing medical records and holding telephone interviews to determine the impact of RAMI on long-term prognosis. Development of hard events was defined as a composite of all-cause death and repeat nonfatal AMI.

\section{Statistical Analysis}

Data were analyzed using StatView 5.0 software (SAS institute, Cary, NC, USA). Continuous variables are described as mean \pm SD and were compared using Student's t-test (variables with normal distribution) or the Mann-Whitney U-test (variables with asymmetric distribution). Categorical variables are described as frequencies and were compared using the $\chi^{2}$ analysis or Fisher's exact test. Multivariate logistic regression analysis was performed for clinical factors with values of $\mathrm{P}<0.2$ in univariate analysis. KaplanMeier methods and log-rank testing were used to determine hard event-free survival. Statistical significance was established at the $\mathrm{P}<0.05$ level.

\section{Results}

All PCI procedures were successful. Tissue plasminogen activator was administered during PCI to 3 patients because of the large amount of thrombus in the infarct-related artery. Warfarin was administered to 5 patients to inhibit thrombus 


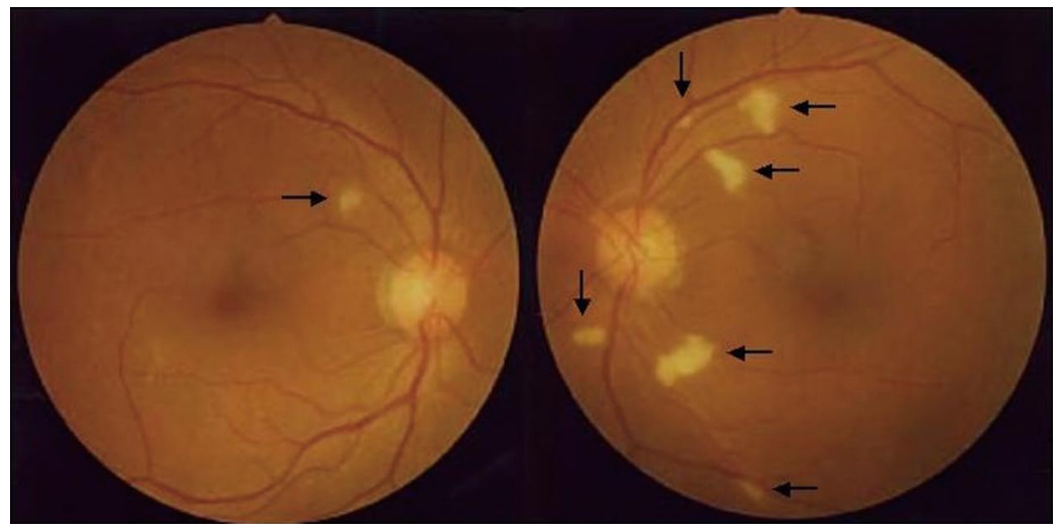

Figure 2. Fundus photographs of both eyes of a 73-year-old man 4 weeks after acute myocardial infarction onset. Soft exudates (arrows) around the optic disc in the both eyes appeared without hemorrhage. He had no history of diabetes mellitus and no retinopathy at the initial examination on day 4. Hemoglobin Alc on admission was 5.2\% and peak creatine kinase was 5,407 IU/L.

Table 1. Clinical Characteristics of Patients With and Without RAMI

\begin{tabular}{|c|c|c|c|}
\hline & RAMI (n=29) & non-RAMI (n=33) & $P$ value \\
\hline Age (years) & $63.0 \pm 8.5$ & $62.7 \pm 11.4$ & 0.906 \\
\hline $\operatorname{Sex}(M / F)$ & $22 / 7$ & $26 / 7$ & 0.999 \\
\hline BMI $\left(\mathrm{kg} / \mathrm{m}^{2}\right)$ & $24.4 \pm 3.6$ & $23.8 \pm 3.4$ & 0.537 \\
\hline Current smoker (\%) & $18(62 \%)$ & $26(79 \%)$ & 0.171 \\
\hline Hypertension (\%) & $21(72 \%)$ & $18(55 \%)$ & 0.191 \\
\hline Diabetes $(\%)$ & $16(55 \%)$ & $7(21 \%)$ & $0.008 *$ \\
\hline Hypercholesterolemia (\%) & $11(38 \%)$ & $14(42 \%)$ & 0.798 \\
\hline \multicolumn{4}{|l|}{ Medications before admission } \\
\hline Anti-diabetic agents & $6(21 \%)$ & $3(9 \%)$ & 0.196 \\
\hline ACEI and/or ARB & $6(21 \%)$ & $6(18 \%)$ & 0.803 \\
\hline Statins & $5(17 \%)$ & $5(15 \%)$ & 0.824 \\
\hline Killip IV (\%) & $2(7 \%)$ & $4(12 \%)$ & 0.676 \\
\hline IRA (RCA/LAD/LCX) & $11 / 3 / 15$ & $12 / 6 / 15$ & 0.674 \\
\hline No. of lesions $(1 / 2 / 3)$ & $18 / 9 / 2$ & $23 / 8 / 2$ & 0.814 \\
\hline Time to reperfusion ( $\min$ ) & $578 \pm 789$ & $791 \pm 1,108$ & 0.392 \\
\hline t-PA $(\%)$ & $3(10 \%)$ & $0(0 \%)$ & 0.108 \\
\hline Thrombectomy (\%) & $21(72 \%)$ & $18(55 \%)$ & 0.191 \\
\hline Stent $(\%)$ & $26(90 \%)$ & $29(88 \%)$ & 0.999 \\
\hline Pre-AMI angina (\%) & $13(45 \%)$ & $15(45 \%)$ & 0.999 \\
\hline Slow flow during PCI (\%) & $3(10 \%)$ & $4(12 \%)$ & 0.999 \\
\hline Reperfusion injury (\%) & $5(17 \%)$ & $4(12 \%)$ & 0.722 \\
\hline $\operatorname{IABP}(\%)$ & $12(41 \%)$ & $8(24 \%)$ & 0.181 \\
\hline Ejection fraction (\%) & $49.9 \pm 13.9$ & $50.3 \pm 10.2$ & 0.889 \\
\hline CRP $(\mathrm{mg} / \mathrm{dl})$ on admission & $0.98 \pm 2.01$ & $0.92 \pm 2.95$ & 0.778 \\
\hline Peak CRP (mg/dl) & $10.0 \pm 5.5$ & $9.7 \pm 4.9$ & 0.794 \\
\hline WBC $(/ \mu 1)$ on admission & $11,370 \pm 4,159$ & $11,676 \pm 3,143$ & 0.743 \\
\hline Peak WBC $(/ \mu \mathrm{l})$ & $12,645 \pm 3,836$ & $12,313 \pm 3,576$ & 0.726 \\
\hline Platelets $\left(\times 10^{4} / \mu 1\right)$ & $26.2 \pm 7.6$ & $26.5 \pm 6.5$ & 0.851 \\
\hline Total cholesterol (mg/dl) & $203 \pm 36$ & $202 \pm 34$ & 0.910 \\
\hline Hemoglobin Alc (\%) & $7.0 \pm 2.0$ & $5.9 \pm 1.4$ & $0.013^{*}$ \\
\hline $\mathrm{BS}(\mathrm{mg} / \mathrm{dl})$ on admission & $197 \pm 80$ & $174 \pm 61$ & 0.210 \\
\hline Peak CK (IU/L) & $3,428 \pm 2,210$ & $2,352 \pm 1,652$ & $0.036^{*}$ \\
\hline Creatinine $(\mathrm{mg} / \mathrm{dl})$ & $0.93 \pm 1.29$ & $0.72 \pm 0.28$ & 0.772 \\
\hline
\end{tabular}

$* \mathrm{P}<0.05$ vs non-RAMI.

RAMI, retinopathy associated with myocardial infarction; BMI, body mass index; ACEI, angiotensin-converting enzyme inhibitor; $\mathrm{ARB}$, angiotensin II receptor blocker; IRA, infarct-related coronary artery; RCA, right coronary artery; LAD, left anterior descending artery; LCX, left circumflex artery; t-PA, tissue plasminogen activator; AMI, acute myocardial infarction; PCI, percutaneous coronary intervention; IABP, intra-aortic balloon pumping; CRP, C-reactive protein; WBC, white blood cell count; BS, blood sugar; $\mathrm{CK}$, creatine kinase.

formation ( 3 cases of large infarct, 2 cases of atrial fibrillation). No in-hospital deaths occurred among the 62 patients.

Diabetic retinopathy was documented on initial fundus examination in 3 of the 62 patients and all 3 patients developed new soft exudates at 4 weeks after AMI onset. New soft exudates appeared in 26 of 59 patients (44\%) who did not display retinopathy at the time of initial fundus examination (Figures 1,2). Overall, new soft exudates around the optic disc developed in $29(47 \%)$ of 62 patients at 4 weeks after AMI onset. Five patients with RAMI experienced ophthalmologic symptoms such as blurred vision and/or metamorphopsia at 4 weeks after the onset of AMI. In contrast, none of the patients without RAMI had ophthalmologic symptoms during the study period.

The frequency of DM, as well as serum levels of $\mathrm{HbAlc}_{1}$ and peak CK (Table 1, univariate analysis), were higher in the RAMI group than in the non-RAMI group. Multivariate analysis showed that DM was an independent predictive factor for the occurrence of RAMI (Table 2). Substituting $\mathrm{HbAlc}$ for the frequency of DM gave very similar results in 
the multivariate analysis. The occurrence of RAMI was significantly greater in patients with DM (16/23: 70\%) than without DM (13/39: 33\%, $\mathrm{P}=0.006$ vs with DM) (Figure 3), although there were no significant differences in pre-AMI medications, such as antidiabetic agents, renin-angiotensin system inhibitors and statins, between the RAMI and nonRAMI groups (Table 1).

In the follow-up study (tracing rate, 97\%; mean tracing term, 52.6 months), hard events developed in 5 patients (allcause death, $n=2$; re-AMI, $n=3$ ) in the RAMI group and 3 patients (all-cause death, $n=2$; re-AMI, $n=1$ ) in the nonRAMI group. No significant difference in the incidence of hard events was seen between groups (Figure 4).

\section{Discussion}

The key findings in the present study are that RAMI developed more frequently in patients with DM compared with those without DM, and that RAMI did not exert any significant effect on long-term prognosis.

DM has been reported to be closely related to systemic inflammation and endothelial dysfunction ${ }^{12,13} \mathrm{We}$ and other investigators have reported that leukocyte entrapment in the retinal capillaries increases and disturbs regional capillary blood flow in animal models of DM!4-16 Even in healthy men, inflammatory mediators released as a consequence of ischemia-reperfusion activate leukocytes, platelets, and endothelial cells in remote organs ${ }^{17}$ Hence, we postulated that extant retinal microvascular dysfunction in DM might be reinforced by systemic inflammation following AMI in itself and ischemia-reperfusion injury. Granger has reported that hypertension and hypercholesterolemia, as well as DM, can exaggerate the microcirculation in vivo elicited by ischemia-reperfusion. ${ }^{18}$ Although we could not confirm a relationship between RAMI and risk factors for atherosclerotic disease other than DM, the degree of microvascular retinal dysfunction in DM might be greater than that among patients with other risk factors before and after ischemiareperfusion.

Soft exudates in the fundus photographs were transient, small and whitish opacities in the retina, and thought to have arisen from pathological accumulation of edematous damaged nerve axis fibers resulting from micro-infarctions of small retinal arterioles or capillaries $!^{19}$ In fact, we have documented by fluorescein angiography that non-perfused areas were consistent with the area of soft exudates in patients with RAMI8 Soft exudates occur frequently in diabetic retinopathy, hypertensive retinopathy and other systemic diseases or condition such as anemia, collagen diseases, HIV infection, and interferon treatment. However, RAMI can be easily differentiated from these other forms of retinopathy by the other changes that commonly coexist with cotton wool spots and by the unique time course from AMI onset? Although PCI-induced microembolic retinopathy has been reported in patients without $\mathrm{AMI}_{2}^{20,21}$ retinopathy did not develop in 10 patients following stent implantation for stable angina in our previous study? Therefore, PCI-related embolism probably does not represent an important contributor to RAMI pathogenesis. The results of the present study with a limited number of subjects show that RAMI did not affect the long-term hard-event prognosis. To clarify the clinical significance of RAMI, large-scale studies are warranted in the fields of both cardiology and ophthalmology.
Table 2. Multivariate Logistic Regression Analysis for RAMI

\begin{tabular}{llc}
\hline & \multicolumn{1}{c}{ OR $(95 \% \mathrm{CI})$} & P value \\
\hline Thrombectomy & $2.573(0.64-10.42)$ & 0.186 \\
IABP & $0.573(0.09-3.58)$ & 0.551 \\
Hypertension & $1.813(0.49-6.76)$ & 0.375 \\
Current smoking & $0.261(0.06-1.11)$ & 0.069 \\
Peak CK & $1.000(1.00-1.01)$ & 0.063 \\
Diabetes & $6.598(1.68-25.90)$ & 0.007 \\
\hline
\end{tabular}

OR, odds ratio; CI, confidence interval. Other abbreviations see in Table 1.

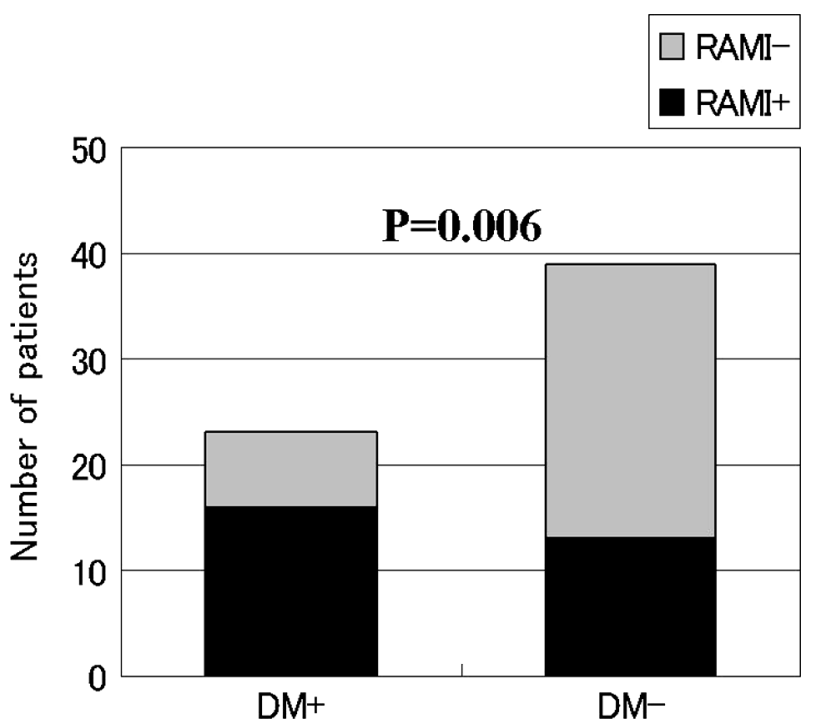

Figure 3. Frequency of retinopathy associated with acute myocardial infarction (RAMI) was significantly greater in patients with diabetes mellitus (DM) than in those without DM.

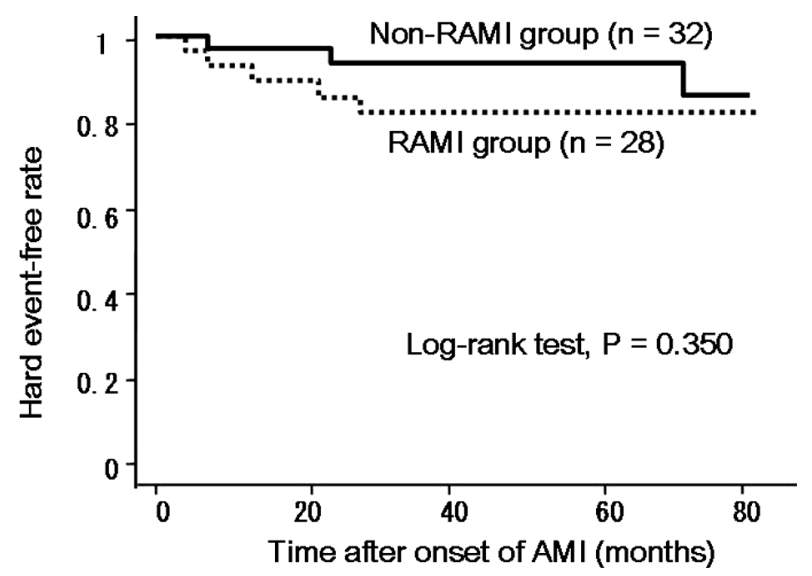

Figure 4. Kaplan-Meier estimate of hard events (all-cause death and repeat nonfatal acute myocardial infarction (AMI)) in patients with and without retinopathy associated with AMI (RAMI). No significant differences in long-term hard events were seen between the RAMI and non-RAMI groups.

\section{Study Limitations}

Firstly, pan-microvascular dysfunction can also be induced by AMI. Thus, whether AMI alone or AMI+ PCI therapy has a greater impact on the occurrence of RAMI is unclear. Conducting a randomized study to compare PCI- and nonPCI-treated patients with AMI may not be ethically acceptable. Secondly, because only 3 of the patients had diabetic 
retinopathy before AMI, the frequency of RAMI in patients with diabetic retinopathy and the influence of RAMI on the progress of diabetic retinopathy should be explored in the future. Thirdly, we did not measure fasting insulin levels, and so could not evaluate insulin sensitivity, which might be related to inflammation and endothelial dysfunction?22,23 It is possible that insulin resistance, as well as DM, is involved in the occurrence of RAMI. Fourthly, 28 patients had funduscopy only without photos at baseline examination. Although funduscopy is suitable for observation in the larger field of the fundus, rather than photography, this difference between methods seems not to have affected detection of RAMI caused by the occurrence of soft exudates around optic disc. Finally, the time between the onset of AMI and reperfusion by PCI was relatively long, because most patients were referred to us from other medical institutions.

In conclusion, DM should be considered as an independent risk factor for RAMI after reperfusion therapy for AMI.

\section{Acknowledgments}

We thank Professors Wilfred Y. Fujimoto and Shinichiro Ueda for their helpful comments regarding the present results, and Sachimi Jinbo for her technical assistance with the data analysis.

\section{References}

1. Frangogiannis NG, Smith CW, Entman ML. The inflammatory response in myocardial infarction. Cardiovasc Res 2002; 53: 31-47.

2. Verma S, Fedak PW, Weisel RD, Butany J, Rao V, Maitland A, et al. Fundamentals of reperfusion injury for the clinical cardiologist. Circulation 2002; 105: 2332-2336.

3. Neumann FJ, Ott I, Gawaz M, Richardt G, Holzapfel H, Jochum M, et al. Cardiac release of cytokines and inflammatory responses in acute myocardial infarction. Circulation 1995; 92: 748-755.

4. Kohsaka S, Menon V, Lowe AM, Lange M, Dzavik V, Sleeper LA, et al; SHOCK Investigators. Systemic inflammatory response syndrome after acute myocardial infarction complicated by cardiogenic shock. Arch Intern Med 2005; 165: 1643-1650.

5. Keren A, Klein J, Stern S. Adult respiratory distress syndrome in the course of acute myocardial infarction. Chest 1980; 77: 161-164.

6. Kusano Y, Endo S, Mukai S, Yamaguchi T. Retinopathy after percutaneous transluminal coronary angioplasty and stent insertion for acute myocardial infarction. Am J Ophthalmol 2003; 136: 557-560.

7. Katayama T, Yasu T, Kinoshita N, Kakehashi A, Ueba H, Hashimoto $\mathrm{S}$, et al. Transient retinopathy after acute myocardial infarction treated by reperfusion therapy: Clinical background and manifestation. J Cardiol 2003; 42: 23-28 (in Japanese).
8. Kinoshita N, Kakehashi A, Yasu T, Katayama T, Kuroki M, Tsurimaki $\mathrm{Y}$, et al. A new form of retinopathy associated with myocardial infarction treated with percutaneous coronary intervention. Br J Ophthalmol 2004; 88: 494-496.

9. Ikeda N, Yasu T, Kubo N, Hashimoto S, Tsuruya Y, Fujii M, et al. Nicorandil vs isosorbide dinitrate as adjunctive therapy to direct balloon angioplasty in acute myocardial infarction. Heart 2004; 90: $181-185$.

10. The TIMI Study Group. The Thrombolysis in Myocardial Infarction (TIMI) trial. N Engl J Med 1985; 312: 932-936.

11. Ikeda N, Yasu T, Kubo N, Nakamura T, Sugawara Y, Ueda S, et al. Daily exercise and bone marrow derived $\mathrm{CD} 34^{+} / 133^{+}$cells after myocardial infarction treated by bare metal stent implantation. Circ J 2008; 72: 897-901.

12. Pradhan AD, Manson JE, Rifai N, Buring JE, Ridker PM. C-reactive protein, interleukin 6 , and risk of developing type 2 diabetes mellitus. JAMA 2001; 286: $327-334$.

13. Meigs JB, Hu FB, Rifai N, Manson JE. Biomarkers of endothelial dysfunction and risk of type 2 diabetes mellitus. JAMA 2004; 291: $1978-1986$.

14. Kinoshita N, Kakehashi A, Inoda S, Itou Y, Kuroki M, Yasu T, et al. Effective and selective prevention of retinal leukostasis in streptozotocin-induced diabetic rats using gliclazide. Diabetologia 2002; 45: $735-739$.

15. Miyamoto K, Hiroshiba N, Tsujikawa A, Ogura Y. In vivo demonstration of increased leukocyte entrapment in retinal microcirculation of diabetic rats. Invest Ophthalmol Vis Sci 1998; 39: 2190-2194.

16. Kim SY, Johnson MA, McLeod DS, Alexander T, Hansen BC, Lutty GA. Neutrophils are associated with capillary closure in spontaneously diabetic monkey retinas. Diabetes 2005; 54: 1534-1542.

17. Kharbanda RK, Peters M, Walton B, Kattenhorn M, Mullen M, Klein $\mathrm{N}$, et al. Ischemic preconditioning prevents endothelial injury and systemic neutrophil activation during ischemia-reperfusion in humans in vivo. Circulation 2001; 103: $1624-1630$.

18. Granger DN. Ischemia-reperfusion: Mechanisms of microvascular dysfunction and the influence of risk factors for cardiovascular disease. Microcirculation 1999; 6: 167-178.

19. Albert DM, Jakobiec FA. Principle and practice of ophthalmology. WB Saunders; 1994; 27-28.

20. Bucci FA Jr, Dimitsopulos TM, Krohel GB. Branch retinal artery occlusion secondary to percutaneous transluminal coronary angiography. Br J Ophthalmol 1989; 73: 309-310.

21. Teitelbaum BA. Asymptomatic unilateral microembolic retinopathy secondary to percutaneous transluminal coronary angioplasty. $J \mathrm{Am}$ Optom Assoc 1999; 70: 587-592.

22. Kim JA, Montagnani M, Koh KK, Quon MJ. Reciprocal relationships between insulin resistance and endothelial dysfunction: Molecular and pathophysiological mechanisms. Circulation 2006; 113: 1888 1904.

23. Ishikawa S, Kayaba K, Gotoh T, Nakamura Y, Kajii E. Metabolic syndrome and C-reactive protein in the general population: JMS Cohort Study. Circ J 2007; 71: 26-31. 